\title{
A Multi-Subpopulation PSO Fusion based Optimal Tuning of PID Controller
}

\author{
Ahmed E. Abdalla \\ Electrical Engineering Dept. \\ Military Technical College Cairo, Egypt
}

\begin{abstract}
In conduct the design problem optimization algorithms, particle swarm optimization (PSO) could be conceivably stuck at a local minimum in a non-proper region of the search. This led to the need of developing a new class of solution method that can overcome this deficiency. For boots out such problems, this paper presents a fusion algorithm of a multisubpopulation particle swarm optimization (MS-PSO). The main idea lies in dividing the main search space into multisubpopulation regions. The fusion is based on performance measurements of the individuals of these multisubpopulations for finding the optimal solution. The results are obtained by testing the particle swarm optimization and multi-subpopulation particle swarm optimization on the tuning of PID controller to a given system to improve its step response parameters. The result is compared with the performance of PID controller tuned using conventional methods. The proposed PSO based PID controller has significant improved performance.
\end{abstract}

\section{General Terms}

Intelligent Control, Optimal Control.

\section{Keywords}

PID, Step Response, Particle Swarm Optimization (PSO), Data Fusion, Multi-Subpopulation.

\section{INTRODUCTION}

Particle swarm optimization (PSO) is an experiential global optimization method which is based on swarm intelligence. PSO technique was invented in the mid-1990s. It comes from the study on the fish and bird group movement actions. The PSO is considered as one of the auspicious optimization techniques [1-3]. The algorithm is widely used and rapidly developed for its easy implementation and few particles required to be tuned. A comprehensive description of the PSO algorithm and the implication of the different parameters of its update rule is given in [ 4 ]. Swarm intelligence is considered as a family of decentralized stochastic algorithms enthused by the conduct of swarms [5]. Comparing with other intelligent algorithms, PSO algorithm has less variables to adjust and is easy to achieve, so it is widely used in engineering [6]. In addition, PSO is employed for finding stable reduced order models of large-scale linear interval systems [7]. The PSO algorithm is used in many trends, controller design, image processing, pattern recognition, finding some threshold for classification problems, and data mining [8-10]. Moreover, PSO is applied in the area of electric power systems to minimize the real power losses of an electric power grid [11]. Interesting application in electrical distribution systems assuming uncertainty in load demand incorporating distributed generation is developed as a multi-objective planning. Two objectives in system planning. The first, is the cost minimization of total installation and operation. The second, is the lowest risk factor [12]. Also, PSO is applied in medical image processing to improve the efficiency of medical image segmentation [13]. However, like many other optimization algorithms such as $[8,9]$, there exist two common drawbacks of using PSO that foil it to find the best optimal solution. First, is when the individual good solutions pull in the population too rapidly. It would cause the population to converge on a suboptimal solution. The second one, refers to too much diversity that may be detrimental to the success of the algorithm. To boot out such two problems, this paper presents a MS-PSO fusion based optimal tuning PID controller. The proposed fusion algorithm relates to apply the PSO algorithm to multi-subpopulation which their union represents the whole population. This search way, help to avoid from getting locked in a suboptimal solution. It goes with two processes. Frist, is partition the main population into some number of subpopulations to search over different particular domain of the assigned population. The second, is to fuse amid the best particles selected from the subpopulations by selecting the particle with the best fitness. The performance of MS-PSO compared to PSO with respect to the performance measurements of dynamical response of the controlled system is validated. This paper is organized as follows: Section-1 gives an introduction to different applications of using PSO algorithm and section-2 introduces the details of the PSO algorithm. While, section-3 explore the controlled system performance indices and section-4 gives the details of the proposed MS-PSO algorithm. Moreover, section-5 explore the simulation result of a practical controlled system and section- 6 explore the benefits of the proposed MS-PSO algorithm compared with classical tuning of PID and standard PSO algorithm. Finally, section-7 reconnoiter the conclusion of the proposed work.

\section{PSO ALGORITHM}

In PSO algorithm, the collection of particles in search space purpose to optimize a fitness function, inspired by the observation of social interaction and animal behaviors such as fish schooling and bird flocking inspiring by the movement of flocks of fish schooling or bird flocking [5]. PSO relies on the exchange of data between particles of the population called swarm. The particles are sited randomly in search space, and they evaluate their quality or fitness at that position. Then, for a predestine number of iterations, each particle moves to a new location which bounces improved fitness than the forgoing position. This new position is gained based on the history of particles own best and current positions with those of the best positions got by other particles in the swarm, with around random perturbations. Thus, in consequent iterations the swarm attains the most optimum solution to the fitness function in the defined space, with a definite number of particles functioning together [6]. The fitness or objective function in PSO algorithm is a performance evaluation measure that is determined by the application field of the algorithm. The performance criterion is usually defined by a mathematical formulation to measure the system performance attained through a performance index. The basic particle swarm optimization algorithm consists of a swarm of "Np" particles, and the position of each particle represents a 
possible solution of the fitness function in M- dimensional search space [9].

The speed and position of each of the particles in a swarm of PSO algorithm, updates by the following equations:

$\vartheta_{i m}^{k+1}=w \vartheta_{i m}^{k}+c_{1} r_{1}^{k}\left(\right.$ pbest $\left._{i m}^{k}-x_{i m}^{k}\right)+c_{2} r_{2}^{k}\left(\right.$ gbest $t_{m}^{k}-$

$\left.x_{i m}^{k}\right)$

$x_{i m}^{k+1}=x_{i m}^{k}+\vartheta_{i m}^{k+1}$

Where,

$x_{i m}^{k}, \vartheta_{i m}^{k}$, pbest $_{i m}^{k}$, and $g b e s t_{m}^{k}$ denotes represents position, velocity, personal best position, and global best position of ith particle of $\mathrm{Np}$ particles at m-dimension out of $\mathrm{M}$ dimensions in kth iteration respectively.

$w$ Represents the inertial weight attached to the particles in advances attained position.

$c_{1}, c_{2}$ Represent acceleration constants.

$r_{1}^{k}, r_{1}^{k}$ Represent random numbers in the range of $[0,1]$.

The velocity update in PSO contains three terms [10]:

* Momentum " $w \vartheta_{i m}^{k}$ "where it signifies the tendency of particle to move in the same direction as it was moving in the previous iteration. It incorporates the effect of previous velocity on current velocity of the particle.

* Cognitive part " $c_{1} r_{1}^{k}\left(p b e s t_{i m}^{k}-x_{i m}^{k}\right)$ " where it signifies the pull to particle's velocity towards its own personal best. Referred to as "memory", "self-knowledge" or "remembrance".

* Social part " $c_{2} r_{2}^{k}\left(\right.$ gbest $\left.t_{m}^{k}-x_{i m}^{k}\right)$ " where it signifies the pull to particle's velocity towards swarm's best. Referred to as "cooperation", "social knowledge" or "shared information". Fig. 1 shows the chart that expresses the procedures of a basic PSO algorithm.

\section{PERFORMANCE INDICES FOR THE PSO ALGORITHM}

The controller performance can be estimated using an objective function based on the error criterion. The following functions are the most conjoint and used in this work:

\section{i. Integral of Absolute Errors (IAE) criterion}

In IAE, the error criterion can be calculated using the following equation:

$I_{I A E}=\int_{0}^{T_{S}}|e(t)| d t$

ii. Integral Square of Errors (ISE) criterion.

In ISE, the error criterion can be calculated using the following equation:

$$
I_{I S E}=\int_{0}^{T_{s}} e(t)^{2} d t
$$

iii. Integral of Time multiplied by Absolute Errors (ITAE) criterion

In ITAE, the error criterion can be calculated using the following equation:

$I_{\text {ITAE }}=\int_{0}^{T_{s}} t|e(t)| d t$

Where, $T_{S}$ is the predictable settling time.

\section{A MS-PSO BASED OPTIMAL TUNING OF PID CONTROLLER ALGORITHM}

In the population based evolutionary algorithms, untimely convergence of population is the most conjoint problems is concerned. In the practical engineering application of evolutionary algorithms, it is a very important research direction on how to avoid individual falling into the local optimum and its untimely convergence. Impose a certain diversity in population will lead to improve the exploration capability during the process of development. In this work paper he proposed algorithm relates to apply the fusion model of algorithms with PSO. It concerns with two processes: one is dividing a particle population into some number of subpopulations to search over several different domains of a particle space; the other is using a fusion mechanism of the global best particles selected from the different subpopulations. This is done by selecting the particle with the best fitness among the set of global best of different subpopulations. The MS-PSO processes are carried out as demarcated in Fig. 2.

\section{SIMULATION AND EXPERIMENTAL RESULTS}

An example of a high-performance drilling process [14] is used to explore the effectiveness of the proposed multisubpopulation PSO algorithm. The drilling process is modeled as a third-order system given by the transfer function described by equation (6), where $s$ is the Laplace operator, $f$ is the command feed, and $\mathrm{F}$ is the cutting force.

$H_{p}(s)=\frac{F(s)}{f(s)}=\frac{1958}{s^{3}+17.89 s^{2}+103.3 s+190.8}$

In order to explore out the control system performance, the simulation studies are implemented, in Matlab/Simulink. The tuning of PID controller is adjusted using the classical method Ziegler-Nichols, direct application of the standard PSO algorithm with population size $=100$ and number of iterations $=100[14]$, and the proposed multi-subpopulation PSO method. PID controller was formed based upon the respective parameters, subpopulation size, $\mathrm{Ns}=8$, population size, $\mathrm{N}_{\text {pop }}=25$, and two reduced number of iterations, Nitr $=50$ and $\mathrm{N}_{\text {itr }}=25$. Table 1 shows the PID controller parameters. The step response of the drilling-force signal is shown in Fig. 3.

\section{RESULTS AND COMPARISION}

Analysis reflects that the design of proposed controller gives a higher robustness and stability. Simulation results show performance improvement in time domain specifications for a step response. Comparison of time domain specifications using the classical method Ziegler-Nichols, direct application of the standard PSO algorithm, and the proposed multisubpopulation PSO method is given by table-3. The proposed MS-PSO algorithm does not require a large number of population in each subpopulation and with lower number of iterations a better performance can be obtained. It reflects that the proposed MS- PSO based controller drastically reduces the overshoot by a large value. Moreover, a comparison for the best performance indices ITAE, IAE, and ISE are obtained for all configuration of the PID controllers are given in Table -3 which ensure the advantage of the proposed controller. 
Table-1. PID controller parameters

\begin{tabular}{|c|c|c|c|c|}
\hline \multirow{2}{*}{\multicolumn{2}{|c|}{ Tuning method }} & \multicolumn{3}{|c|}{ PID Controller Parameters } \\
\hline & & $\mathbf{P}$ & $\mathbf{I}$ & D \\
\hline \multicolumn{2}{|r|}{ Ziegler-Nichols } & 0.5128 & 1.6649 & 0.0395 \\
\hline \multicolumn{2}{|r|}{ Standard PSO [12] } & 0.7164 & 1.4665 & 0.0984 \\
\hline \multirow{2}{*}{$\begin{array}{l}0 \\
\mathscr{n}_{2} \\
\tilde{n}^{\prime} \\
\sum^{2}\end{array}$} & Npop $=50$, Nitr $=25$ & 0.4452 & 1.1091 & 0.1075 \\
\hline & Npop $=25$, Nitr $=25$ & 0.4066 & 0.9787 & 0.1154 \\
\hline
\end{tabular}

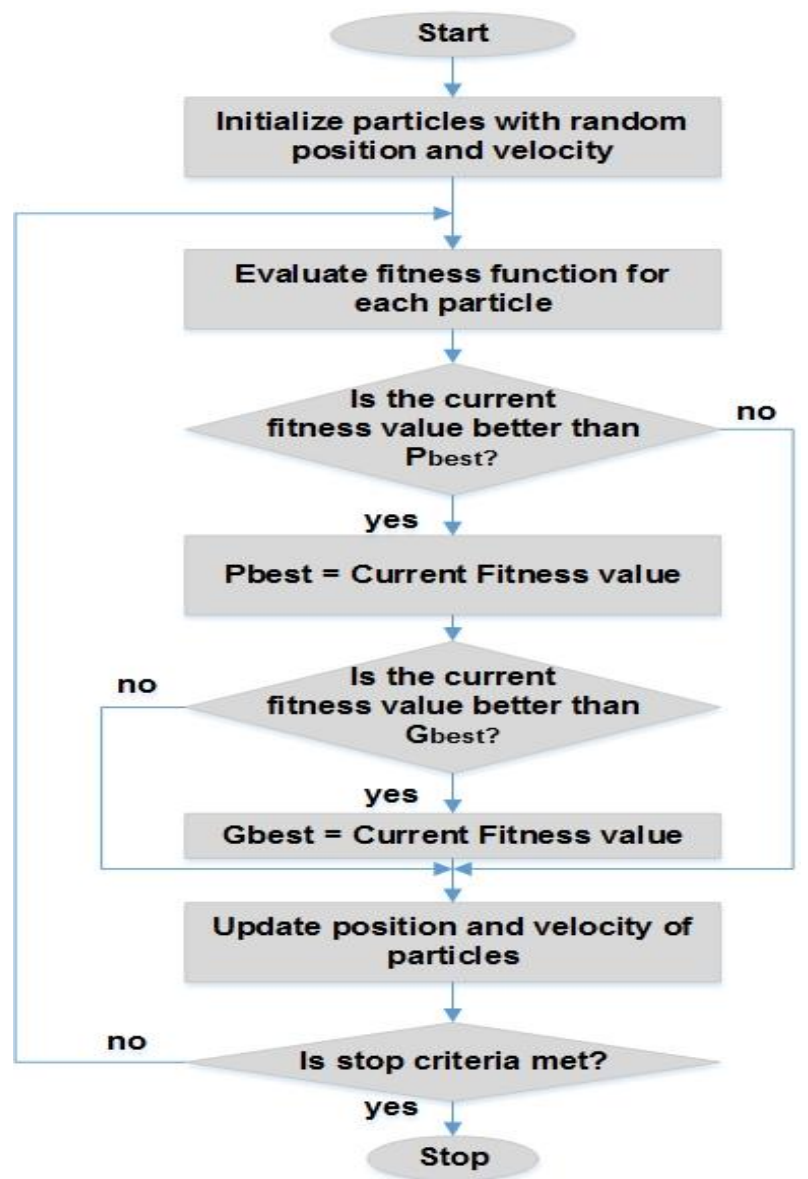

Fig 1: Chart of a basic PSO algorithm.

Table-2. Comparison of time domain specifications

\begin{tabular}{|c|c|c|c|c|}
\hline \multirow{2}{*}{\multicolumn{2}{|c|}{ Tuning method }} & \multicolumn{3}{|c|}{$\begin{array}{l}\text { Time Domain } \\
\text { Specifications }\end{array}$} \\
\hline & & $\begin{array}{l}\text { Rise } \\
\text { Time }\end{array}$ & $\begin{array}{l}\text { Sett. } \\
\text { Time }\end{array}$ & PO \\
\hline & Ziegler-Nichols & 0.1470 & 1.6069 & $42.34 \%$ \\
\hline & Standard PSO [12] & 0.1055 & 0.5923 & $22.82 \%$ \\
\hline O & $\mathrm{N}_{\mathrm{pop}}=50, \mathrm{~N}_{\mathrm{itr}}=25$ & 0.1191 & 0.5752 & $6.29 \%$ \\
\hline$\overline{\tilde{n}}$ & $\mathrm{~N}_{\mathrm{pop}}=25, \mathrm{~N}_{\mathrm{itr}}=25$ & 0.1175 & 0.6461 & $3.63 \%$ \\
\hline
\end{tabular}

Table-3. Comparison of performance index

\begin{tabular}{|c|c|c|c|c|}
\hline \multicolumn{2}{|r|}{ Tuning method } & ITAE & IAE & ISE \\
\hline \multicolumn{2}{|r|}{ Ziegler-Nichols } & 0.1040 & 0.1463 & 0.2973 \\
\hline \multicolumn{2}{|r|}{ Standard PSO [12] } & 0.0309 & 0.0828 & 0.1595 \\
\hline \multirow{2}{*}{$\begin{array}{l}0 \\
\tilde{2} \\
\tilde{n}^{\prime} \\
\Sigma\end{array}$} & Npop $=50$, Nitr $=25$ & 0.0256 & 0.0700 & 0.1266 \\
\hline & Npop $=25$, Nitr $=25$ & 0.0282 & 0.0691 & 0.1303 \\
\hline
\end{tabular}

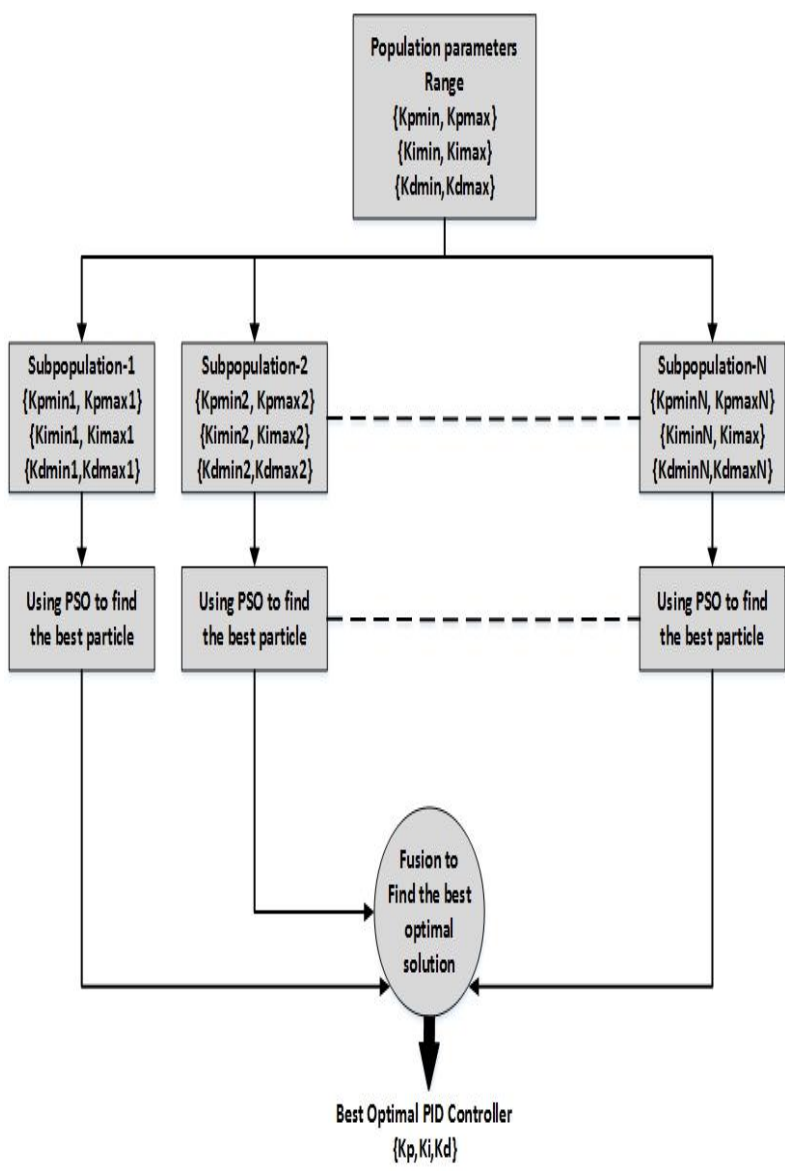

Fig. 2: The chart that expresses the processes of MS-PSO algorithm

\section{CONCLUSIONS}

In this paper a fusion algorithm of MS-PSO has been introduced. It is shown analytically and graphically that there is a substantial improvement in the time domain specification in terms of a lower overshoot with comparable rise time and settling time compared with the existing traditional techniques of tuning the controller. The performance indices for different error criterions; ITAE, IAE, and ISE; have is proved to be the lowest using the proposed controller. Moreover, it can be concluded that with a lower number of total population in subpopulations with lower iterations number would result in a better PID controller time domain specifications and lowest performance index for different error criterions. Parallel computing can be set up to optimize of the time of PID tuning which can be reflected in real time applications 


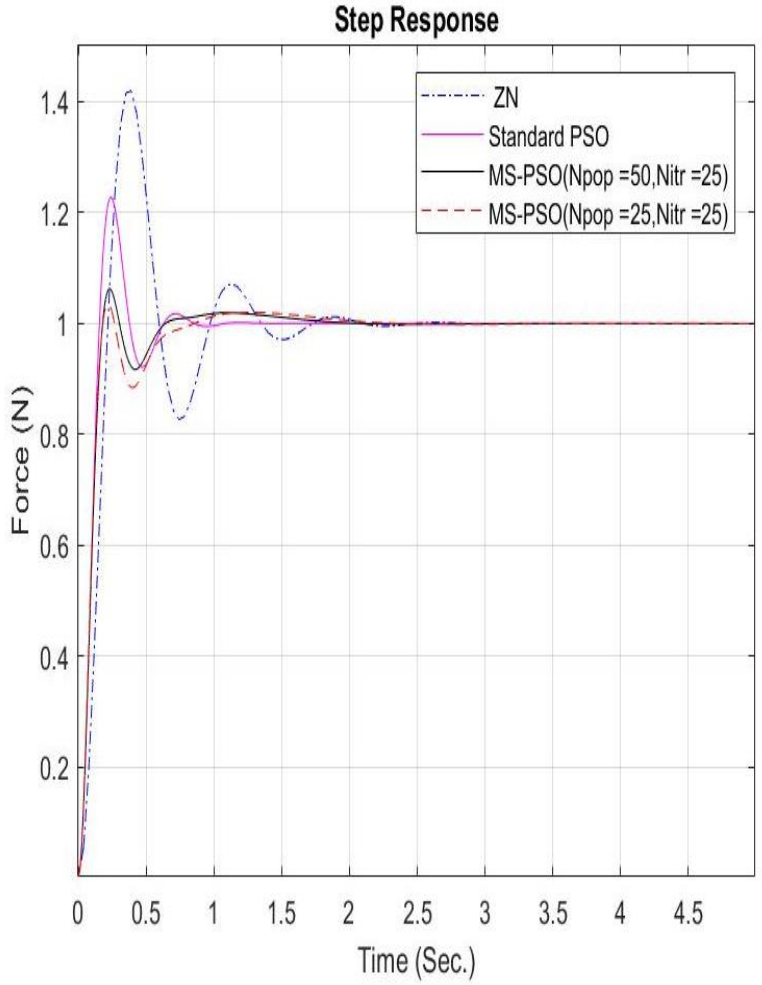

Fig 3: Unit step response with Z-N controller, standard PSO based controller, and MS-PSO based controller

\section{REFERENCES}

[1] J. Kennedy, and R.C. Eberhart, Swarm Intelligence. Morgan Kaufmann Publishers, Inc., CA, USA, 2001.

[2] R.C. Eberhart, and Y. Shi, "Comparison between Genetic Algorithms and Particle Swarm Optimization", In et al. V. W. Porto, editor, Evolutionary Programming, Vol. 1447 of Lecture Notes in Computer Science, Springer, 1998, pp. 611-616.

[3] M. Jamil and X.Yang, "A literature survey of benchmark functions for global optimization problems", Int. Journal of Mathematical Modelling and Numerical Optimization, Vol. 4, No. 2, 2013, pp. 150-194.

[4] M. Juneja, S. K. Nagar, "Particle swarm optimization algorithm and its parameters: A review", International Conference on Control Computing Communication and Materials (ICCCCM), 2016, pp. 1-6.
[5] X. Zhang, W. Hu, S. Maybank, X. Li, and M. Zhu, "Sequential particle swarm optimization for visual tracking”, IEEE CVPR, 2008, pp. 1-8.

[6] W. Jiang, Y. Zhang, and R. Wang, "Comparative study on several PSO algorithms", The 26th Chinese Control and Decision Conference (CCDC), 2017, pp. 1117 1119.

[7] D. Saini and R. Prasad, "Order reduction of linear interval systems using particle swarm optimization", International Journal of Engineering Science and Technology, Vol. 2, 2010, pp.316-319.

[8] C.L.Lin, H.Y.Jan, Evolutionarily multiobjective PID control for linear brushless DC motor, IEEE Int. Conf. Industrial Elect. 2002, pp. 2033-2038.

[9] Binh Tran and Bing Xue and Mengjie Zhang."A New Representation in PSO for Discretisation-Based Feature Selection", IEEE Transactions on Cybernetics, vol. 48, no. 6,2018 , pp.1733-1746.

[10] M. Ghannad-Rezaie, H. Soltanain-Zadeh, M.-R. Siadat, and KV. Elisevich, "Medical Data Mining using Particle Swarm Optimization for Temporal Lobe Epilepsy", IEEE Congress on Evolutionary Computation, July 2006, pp. 761-768.

[11] M. Jia , L. Chen , X. Yuan, Y. He, and L. Zhao "Sensor configuring optimization for grid harmonic monitoring based on improved PSO algorithm" 2017 IEEE 2nd Advanced Information Technology, Electronic and Automation Control Conference (IAEAC), 2017 pp. 2296 - 2299.

[12] S. Ganguly, N. C. Sahoo, and D. Das, "Multi-objective particle swarm optimization based on fuzzy-Paretodominance for possibilistic planning of electrical distribution systems incorporating distributed generation", Fuzzy Sets and Systems, vol. 213, 2013, pp. 47-73.

[13] Liu Na, Jiang Yan, and Li Shu, "Application of PSO algorithm with dynamic inertia weight in medical image thresholding segmentation", IEEE 19th International Conference on e-Health Networking, Applications and Services (Healthcom), 2017.

[14] S.GirirajKumar, D. Jayaraj, and A.Kishan "PSO based Tuning of a PID Controller for a High Performance Drilling Machine", International Journal of Computer Applications, volume 1 - No. 19, 2010, pp. 12-18. 